\title{
The Perfect Surprise: A New Analysis in Dynamic Epistemic Logic
}

\author{
Leander Vignero ～Lorenz Demey
}

\begin{abstract}
In this article, we present a new logical framework to think about surprise. This research does not just aim to better understand, model and predict human behavior, but also attempts to provide tools for implementing artificial agents. Moreover, these artificial agents should then also be able to reap the same epistemic benefits from surprise as humans do. We start by discussing the dominant literature regarding propositional surprise and explore its shortcomings. These shortcomings are of both an empirical and a conceptual nature. Next, we propose a philosophical solution to the problems that ail these systems, based on the notion of issue of epistemic interest. epistemic interest. Finally, we give a formal framework to think about surprise. More specifically, we develop a probabilistic dynamic epistemic logic (called $\mathcal{S U R \mathcal { P }} \mathcal{R} \mathcal{I S E}$ !) that succeeds at formalizing the relevant philosophical concepts. This will be done through an issue-management system grounded topology. As an added bonus, the additional expressive power allows us to capture a richer variety of scenarios, and it also enables a more careful analysis of said scenarios.
\end{abstract}

\section{Introduction}

Surprise is a familiar emotion that permeates everyday life. The range of surprising events is endless: a hot day in Iceland, stock market crashes, a coffee bar that does not serve cappuccinos, a vegetarian butcher... Despite this familiarity, it is a quirky phenomenon. This makes reasoning about surprise a tricky matter. The applications of a good understanding of surprise seem just as endless: modeling and predicting human behavior, applications in machine learning... We want to make clear that in this article, we are only interested in the surprise elicited by propositional content: systems that investigate sensory surprise like that of Baldi and Itti will not be at issue here [BI05, BI10, IB09.

In applied logic there is the earlier work of Demey [Dem15]. The logical

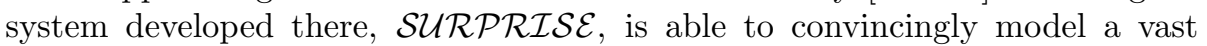
array of situations. It is based on ideas by the computer scientists Macedo and Cardoso [MC01, who equate surprise with unexpectedness. Most people share the intuition that surprise is somehow related to unexpectedness Cha64, OP87, SPMR95. Indeed, highly likely events are never experienced 
as surprising, and for this reason many philosophers and computer scientists have tried to incorporate this in their formalizations. Yet, we will argue in this paper that there is more to surprise than mere unexpectedness. Indeed, there seems to be a class of pathological scenarios that cannot be accurately modeled by $\mathcal{S U R \mathcal { P }} \mathcal{R} \mathcal{I} \mathcal{E}$. For this reason, we will develop a new logic (called $\mathcal{S U R P \mathcal { R }} \mathcal{I S E}$ !) that is able to tackle these problems, thereby allowing us to capture a wide range of dynamic-epistemic situations more accurately. As an added bonus, many of the ideas we develop are well-suited for other applications in probabilistic dynamic epistemic logic [DK15, DS15].

The strategy for crafting $\mathcal{S U R \mathcal { P }} \mathcal{I} \mathcal{S E}$ ! is simple: we look at how human agents act upon learning surprising information. This means that in order to harness the full epistemic potential of surprise, it is worth looking at what cognitive science has to say about this aspect of human cognition. Specifically, we will look at the work of the psychologist Charlesworth Cha64. Note that this strategy is particularly interesting if one wants to make their system use surprise in a way that is similar to the way human agents use it.

The remainder of this article is organized as follows. In Section 2 we briefly describe some of the earlier formal systems of surprise, and discuss the problems that those systems face. Next, in Section 3, we employ the strategy described above. Looking at the cognitive science of surprise, we introduce the central notion of this paper, viz. issues of epistemic interest. In Section 4 we put this

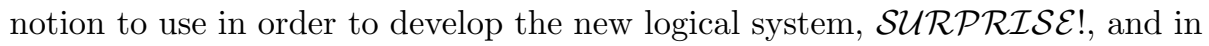
Section 5 we establish some fundamental metalogical properties of this system. In Section 6 we illustrate the expressive power of $\mathcal{S U R \mathcal { P }} \mathcal{R} \mathcal{I} \mathcal{E}$ !, by showing how this system can naturally capture a broad range of interesting scenarios. Finally, we conclude in Section 7 and mention some questions for further research.

\section{Previous Formal Systems of Surprise}

In this section we briefly describe some of the earlier formal systems of surprise, and discuss the problems that those systems face. Logicians and computer scientists are not primarily interested in the phenomenology of surprise, yet they have incorporated one important aspect of human surprise in their formal models, viz. the fact that surprise comes in degrees [MC01, MRC05]. This fact is also well-documented in cognitive science [Rei00, SPMR95].

As mentioned above, we will mainly focus on Demey's formalization Dem15 of the ideas of Macedo and Cardoso MC01. In view of the close association between surprise and unexpectedness, these authors define the surprise elicited by an event $X$ in an agent $a$ as inversely correlated with the probability that $a$ ascribed to $X$. Formally, this is captured by means of a surprise intensity function, which maps events to the intensity of surprise that they elicit! The simplest surprise intensity function looks as follows: $S(X)=1-P(X)$, where $S(X)$ is the surprise elicited by $X$ in $a$, and $P(X)$ is the (subjective) probability that $a$ ascribed to $X$. For example, hot weather in Iceland is highly

\footnotetext{
${ }^{1}$ All surprise intensities in this article will be rational values between 0 and 1.
} 
unexpected (e.g. $P(X)=0.01$ ), so if this actually occurs, it will be experienced as highly surprising (in casu, $S(X)=1-P(X)=1-0.01=0.99$ ). By contrast, hot weather in the Sahara desert is completely in line with our expectations (e.g. $P(X)=0.98$ ), so if this actually occurs, it will elicit hardly any surprise (in casu, $S(X)=1-P(X)=1-0.98=0.02$ ).

It turns out, however, that this framework is insufficient to adequately model all cases of surprise. Consider the following scenario: one randomly picks a ball from a bin containing 10000 numbered balls, say ball 4592. The probability of picking this ball is $\frac{1}{10000}$, and hence, the surprise intensity elicited by picking this ball is predicted to be $1-\frac{1}{10000}=\frac{9999}{10000}$, i.e. we are predicted to experience a very high degree of surprise. This prediction does not square with our intuitions about this scenario: intuitively, nobody would be surprised upon picking ball 4592 from the bin (rather than, say, ball 4591, ball 4593, or any other ball). Macedo and Cardoso have acknowledged this problem for their framework [MRC05, and have proposed an alternative MCRL09, MC12, MC17. However, this alternative framework unfortunately turns out to be incompatible with the compositional semantics of propositional calculus: for example, the surprise intensity of a disjunction could take on negative values, which is clearly nonsensical.

Another problem is that the older systems seriously lack in expressive power. Surprise occurs in dynamic situations: something has to happen before one can feel surprise. Demey already highlighted this dynamic component to surprise, and therefore used a system of dynamic epistemic logic to model it Dem15. More specifically, his system $\mathcal{S U} \mathcal{R} \mathcal{P} \mathcal{R} \mathcal{I} \mathcal{E}$ is an extension of public announcement logic, in which the occurrence of an event is formalized by means of a public announcement operator. For instance, letting $p_{\text {hotice }}$ be the proposition that it is hot in Iceland, the expression

$$
S\left(p_{\text {hotice }}\right)=0 \wedge\left[! p_{\text {hotice }}\right] S\left(p_{\text {hotice }}\right)=0.99
$$

should be read as follows: initially (i.e. before any information has been arrived), the agent does not feel any surprise about it being hot in Iceland, but then the information that it is hot in Iceland actually arrives, and after learning this new information, the agent is highly surprised. Although this goes a long way toward modeling a broad range of scenarios, there is a large class of problems that is still left out. For example, consider the ball-picking scenario from before, and let $p_{4592}$ be the proposition that expresses that ball 4592 has been picked from the bin. The $\mathcal{S U R \mathcal { P }} \mathcal{R} \mathcal{I} \mathcal{S} \mathcal{E}$ system will make the following prediction:

$$
S\left(p_{4592}\right)=0 \wedge\left[! p_{4592}\right] S\left(p_{4592}\right)=\frac{9999}{10000}
$$

This expression adequately captures the dynamic aspects of surprise, but it still says that after ball 4592 has actually been picked from the bin, the agent will be highly surprised about this. In other words, the formal system still goes completely against our intuitive assessment of this scenario.

Things are even worse than they might first seem. Consider the following variation on the ball-picking scenario. Suppose someone tells you, before any 
ball has actually been picked, that she believes that 4592 is the ball that will be picked. Consequently, a ball is effectively picked from the bin, and it turns out to be indeed ball 4592 . In this scenario, you probably would be very surprised! After all, how could the person know what ball was going to be picked? The $\mathcal{S U R \mathcal { P }} \mathcal{R} \mathcal{I} \mathcal{S} \mathcal{E}$ framework is insufficiently expressive to capture the relevant differences between the original ball-picking scenario and the variation just described.

The new framework to be developed in this paper, $\mathcal{S U R \mathcal { P }} \mathcal{I} \mathcal{I} \mathcal{E}$ !, will not only be able to predict reasonable surprise intensities in both the original ballpicking scenario and the variation (i.e. low surprise in the original scenario versus high surprise in the variation), but it is also sufficiently expressive to enable us to model both scenarios in adequate detail. Specifically, the logic will be able to capture the idea of someone predicting an outcome in advance, through a feature that we will call issue-raising. This is a strengthening of Demey's original philosophical point, because issue-raising is itself a highly dynamic phenomenon.

For the sake of completeness, it should be added that $\mathcal{S U R \mathcal { P }} \mathcal{I} \mathcal{S E}$ is not the only logical system to be inspired by the work of Macedo and Cardoso. There are alternative systems on the market, like those of Lorini and Castelfranchi CL03, LC07, which also draw upon these same ideas. However, as was argued in Dem15, these alternative systems are inadequate, because they do not fully do justice to the dynamic nature of surprise.

\section{The Cognitive Side of Surprise}

The developmental psychologist Charlesworth has investigated the relation between surprising stimuli and curiosity Cha64. It turns out that when children are faced with surprising events, they are more likely to go out and investigate these events, in comparison to when they are faced with novel stimuli. Novelty is itself a form of unexpectedness, and this again indicates that surprise should be distinguished from mere unexpectedness. The main point, however, remains that surprising events prompt investigations into the cause of the surprising event.

Let us now consider this psychological finding from a more philosophical setting: surprising events make us aware of some kind of salient epistemic shortcoming in our assessment of a given situation. This insight also shines through in the truism that great scientific discoveries arise from the observation of surprising data. The main observation here is that surprise intensity seems to factor into some form of interest in events.

Human agents know that some of the hypotheses they have are wrong. They are confronted with large amounts of information whilst having only limited cognitive resources at their disposal to investigate and examine their hypotheses. What they (and artificial agents, for that matter) therefore need, is a mechanism for sifting through this vast amount of information efficiently. Surprise can play this role: it separates the epistemically salient events from the epistemically bland ones. 
A particularly nice way to express this point, involves understanding the surprise intensity function of a rational agent as an epistemic triage system: ceteris paribus, surprise is able to distinguish the most epistemically salient events. The different surprise intensities felt upon encountering different events tell us which events to further investigate, and perhaps even in what order. This can be compared to how nurses perform triage in emergency rooms: they pick out the patients with the most severe and urgent conditions to be treated first.

We therefore introduce the notion of issues of epistemic interest, which is the central philosophical notion of this article. Issues of epistemic interest are precisely those issues that contain information that should be further explored and investigated for the benefit of the agent. (This is not to say that surprise in human agents can never be off the mark, as we will see in the discussion of red herrings in Section 7 , )

Finally, it should be emphasized that similar ideas have also been explored elsewhere in the literature, in particular in the aforementioned work by Lorini and Castelfranchi [LC07. ${ }^{2}$ For example, their definition of mismatch-based surprise involves a representation $\varphi$ that is under the agent's active scrutiny, which is formalized as Test $(\varphi)$. In order for $\varphi$ to come under the agent's active scrutiny, it has to be 'retrieved' first, which is formalized by means of a dynamic operator $[$ retrieve $(\varphi)]$. The crucial axiom is $[\operatorname{retrieve}(\varphi)] \operatorname{Test}(\varphi)$ : after the mental operation of retrieving $\varphi$, the agent is actively scrutinizing $\varphi$. Lorini and Castelfranchi's notion of 'representations under active scrutiny' can be compared to our 'issues of epistemic interest', while their 'retrieving'-operation is similar to our notion of 'issue-raising'. We will revisit this comparison in more detail in Section 4 (cf. Footnote 8).

\section{A New Dynamic Surprise Logic: $\mathcal{S U R \mathcal { R }} \mathcal{R} \mathcal{I} \mathcal{S}$ !}

Based on the notion of 'issue of epistemic interest', we will in this section construct a logical system that can capture the entire range of surprise phenomena described above. First, we describe the syntax for the language $\mathcal{L}$ in Subsection 4.1, and then we discuss its semantics in Subsection 4.2.

\subsection{Syntax}

Let us start start by describing the language $\mathcal{L}$ of the logic $\mathcal{S U R \mathcal { P }} \mathcal{R} \mathcal{I} \mathcal{S}$ !, which consists of a static part and a dynamic part. This language will contain the usual propositional connectives, thus allowing for propositional reasoning. Secondly, since this is a system of epistemic logic, the knowledge operator $K$ will be included in $\mathcal{L}$. We also find inspiration in Bayesianism, specifically the insight that our doxastic attitudes have a probabilistic nature. Reasoning about probabilities requires linear inequalities of probabilities as well. It are these linear inequalities of probabilities that will be included in the language. Of course there is a surprise operator, $S(\cdot)$, and there is also a closure operator, $c l(\cdot)$,

${ }^{2}$ Thanks to an anonymous referee for pointing out this connection. 
which will be discussed later in this section. This concludes the static part of the language. Finally, there are two dynamic operators. The first, [!.]., will allow for the expression of public announcements, while the second, [\#·]., will allow for the expression of issue-raising. All of this is formalized in Definition 1 .

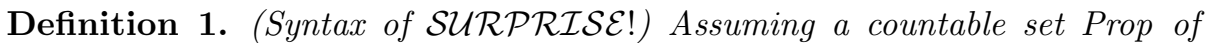
propositional variables, the language $\mathcal{L}$ is defined by the following BNF:

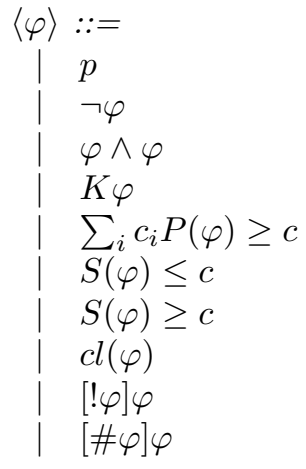

well-formed formulas:
propositional atoms
negation
conjunction
knowledge operator
linear combinations of probabilities
surprise
surprise
closure
public announcement
issue raising

(All numbers $c_{i}, c$ in this BNF are rationals.)

Because of the expressive power of linear combinations, the $\geq$-format suffices to define all probability formulas. In particular, $\sum_{i} c_{i} P\left(\varphi_{i}\right) \leq c$ can be defined as $\sum_{i}\left(-c_{i}\right) P\left(\varphi_{i}\right) \geq-c$, while $\sum_{i} c_{i} P\left(\varphi_{i}\right)=c$ can be defined as the conjunction $\left.\sum_{i} c_{i} P\left(\varphi_{i}\right) \geq c \wedge \sum_{i} c_{i} P\left(\varphi_{i}\right) \leq c\right]^{3}$ By contrast, since we do not consider linear combinations of $S$-values, the formulas $S(\varphi) \geq c$ and $S(\varphi) \leq c$ both need to be taken as primitive. (We can then use the conjunction of these two formulas to define $S(\varphi)=c.)^{4}$

\subsection{Semantics}

We now define the class of relevant models, which will allow us to formulate the semantics for the language $\mathcal{L}$. However, for the sake of comparison, we first provide the definition for the models of Demey's language [Dem15]:

Definition 2. An SP-model is a tuple of the form $\mathbb{M}=\langle W, R, \mu, \sigma, V, \Pi\rangle$, where $W$ is a non-empty, finite set of possible worlds, $R$ is an equivalence relation on $W, \mu$ is a function mapping possible worlds $w$ to their respective probability mass functions $\mu(w): W \rightarrow[0,1]$, which are subject to the following conditions

1. $\forall w, v \in W:$ if $(w, v) \notin R$, then $\mu(w)(v)=0$,

\footnotetext{
${ }^{3}$ And of course, $<-$ and $>$-type probability formulas can be defined as the negations of the corresponding $\geq$ - and $\leq$-type formulas, respectively.

4 Note that this language has many commonalities with that of Demey Dem15; also cf. DK15, DS15]. In fact, the language of $\mathcal{S U R \mathcal { P }} \mathcal{R} \mathcal{I} \mathcal{S}$ ! is a proper extension of that of the original framework $\mathcal{S U R \mathcal { P }} \mathcal{R} \mathcal{I} \mathcal{S} \mathcal{E}$ : only the static closure operator and the dynamic issueraising operator are new.
} 
2. $\forall w \in W: \mu(w)(w)>0$,

$\sigma$ is a function mapping possible worlds $w$ to their respective surprise intensity functions $\sigma(w): \wp(W) \rightarrow[0,1], V:$ Prop $\rightarrow \wp(W)$ is the valuation map, and $\Pi$ is a partition on $W$.

The partition $\Pi$ will be used to give formal meaning to the closure operator $c l(\cdot){ }^{5}$ Furthermore, note that $\mu(w)$ is defined on individual states, and can additively be lifted to sets of states: $\mu(w)(X):=\sum_{x \in X} \mu(w)(x)$, for any $X \subseteq$ $W$. By contrast, $\sigma(w)$ is defined directly on sets of states, so it might happen, for example, that $\sigma(w)(\{x, y\}) \neq \sigma(w)(\{x\})+\sigma(w)(\{y\})$. This technical difference between $\mu$ and $\sigma$ illustrates the underlying conceptual difference between the well-behaved epistemological notion of probability (degree of belief) and the 'raw' psychological notion of (degree of) surprise.

Definition 3. (Partition topology) Let $W$ be a set and $\Pi$ be a partition on $W$. The partition topology $\tau$ is the topology which has the cells of $\Pi$ as a basis. In other words, the partition topology is the natural topology induced by $\Pi$ on $W$ SS78, i.e. $\tau=\{\bigcup \mathcal{X} \mid \mathcal{X} \subseteq \Pi\}$.

One can now easily check that that $\tau$ satisfies the three axioms of topologies:

1. Since $\emptyset, \Pi \subseteq \Pi$, we have $\emptyset=\bigcup \emptyset \in \tau$ and $W=\bigcup \Pi \in \tau$.

2. Any union of opens is again just a union of cells of $\Pi$ or the empty set, and therefore any union of opens is open by definition.

3. Finally, we check that the intersection of two arbitrary opens is again open. If one of the opens is the empty set, we are done. Otherwise we are taking the intersection of two unions of cells of $\Pi$, say $U$ and $V$. Suppose that $x \in U \cap V$. Then $x$ is an element of a cell that is shared by $U$ and $V$. Since we chose $x$ arbitrarily, it now follows that $U \cap V$ is just a union of cells of $\Pi$ and thus open by definition.

Notation 1. $\Pi(\cdot)$ denotes the closure with respect to the partition topology on $W$ induced by $\Pi$, i.e. for any $X \subseteq W$, we have $\Pi(X):=\bigcup\{V \in \Pi \mid X \cap V \neq \emptyset\}$.

Exploiting the natural topological structure of $\Pi$ will allow us to employ insights from topology when reasoning about the semantics of $\mathcal{L}{ }^{6}$ With these concepts in hands, we are now ready to give a semantics for $\mathcal{L}$ :

\footnotetext{
${ }^{5}$ This partition $\Pi$ is the only component that is not present in [Dem15]. Our notion of an SP-model is thus essentially an extension of the notion of surprise model from Dem15.

${ }^{6}$ For example, suppose one wants to check that the axiom $\operatorname{cl}(\operatorname{cl}(\varphi)) \rightarrow \operatorname{cl}(\varphi)$, which we introduce in Section 5.1 holds in all models. All one has to do in order to check this, is rehearsing an all too familiar slogan from topology: the closure of a closed set is itself. There is no need to prove the equivalent statement again for partitions with the closure defined as in Notation 1
} 
Definition 4. (Semantics) The semantics of $\mathcal{L}$ is given by:

\begin{tabular}{|c|c|c|}
\hline $\mathbb{M}, w \models p$ & iff & $w \in V(p)$ \\
\hline $\mathbb{M}, w \models \neg \varphi$ & iff & $\mathbb{M}, w \not \models \varphi$ \\
\hline $\mathbb{M}, w \models \varphi \wedge \psi$ & iff & $\mathbb{M}, w=\varphi$ and $\mathbb{M}, w \models \psi$ \\
\hline $\mathbb{M}, w=K \varphi$ & iff & $\forall v \in W$ such that $w R v: \mathbb{M}, v=\varphi$ \\
\hline $\mathbb{M}, w=\sum_{i} c_{i} P\left(\varphi_{i}\right) \geq c$ & iff & $\sum_{i} c_{i} \mu(w)\left(\llbracket \varphi_{i} \rrbracket^{\mathbb{M}}\right) \geq c$ \\
\hline $\mathbb{M}, w \models S(\varphi) \leq c$ & iff & $\left\{\begin{array}{l}\sigma(w)\left(\llbracket \varphi \rrbracket^{\mathbb{M}}\right) \leq c \text { if } \sigma(w)\left(\llbracket \varphi \rrbracket^{\mathbb{M}}\right) \text { is defined, } \\
c=0 \text { otherwise, }\end{array}\right.$ \\
\hline $\mathbb{M}, w \models S(\varphi) \geq c$ & iff & $\left\{\begin{array}{l}\sigma(w)\left(\llbracket \varphi \rrbracket^{\mathbb{M}}\right) \geq c \text { if } \sigma(w)\left(\llbracket \varphi \rrbracket^{\mathbb{M}}\right) \text { is defined, } \\
c=0 \text { otherwise, }\end{array}\right.$ \\
\hline $\mathbb{M}, w \models c l(\varphi)$ & iff & $w \in \Pi\left(\llbracket \varphi \rrbracket^{\mathbb{M}}\right)$ \\
\hline $\mathbb{M}, w \models[! \varphi] \psi$ & iff & if $\mathbb{M}, w \models \varphi$ then $\mathbb{M} ! \varphi, w \models \psi$, \\
\hline $\mathbb{M}, w \mid=[\# \varphi] \psi$ & iff & $\mathbb{M} \# \varphi, w \models \psi$ \\
\hline
\end{tabular}

For any $\varphi \in \mathcal{L}$, the truth set, $\llbracket \varphi \rrbracket^{\mathbb{M}}$, of $\varphi$ in a model $\mathbb{M}$ is defined to be the set of all possible worlds in $\mathbb{M}$ in which $\varphi$ is true:

$$
\llbracket \varphi \rrbracket^{\mathbb{M}}:=\{w \mid \mathbb{M}, w \models \varphi\} .
$$

The semantic clauses for the $[! \varphi]-$ and $[\# \varphi]$-operators refer to the updated models $\mathbb{M} ! \varphi$ and $\mathbb{M} \# \varphi$. We first define the model updated by public announcement. Let $\mathbb{M}=\langle W, R, \mu, \sigma, V, \Pi\rangle$ and $\varphi \in \mathcal{L}$. Under the assumption that $\llbracket \varphi \rrbracket^{\mathbb{M}} \neq \emptyset$, we define the SP-model $\mathbb{M} ! \varphi=\langle W ! \varphi, R ! \varphi, \mu ! \varphi, \sigma ! \varphi, V ! \varphi, \Pi ! \varphi\rangle$ as follows:

- $W ! \varphi:=\llbracket \varphi \rrbracket^{\mathbb{M}}$,

- $R ! \varphi:=R \cap\left(\llbracket \varphi \rrbracket^{\mathbb{M}} \times \llbracket \varphi \rrbracket^{\mathbb{M}}\right)$,

- $(\mu ! \varphi)(w)(X):=\frac{\mu(w)(X)}{\mu(w)\left(\llbracket \varphi \rrbracket^{\mathbb{M}}\right)}$ for all $w \in W ! \varphi, X \subseteq W ! \varphi$

- $(\sigma ! \varphi)(w)(X):=1-\mu(w)(\Pi(X))$ for all $w \in W ! \varphi$ and all $X \subseteq W ! \varphi$,

- $(V ! \varphi)(p):=V(p) \cap \llbracket \varphi \rrbracket^{\mathbb{M}}$ for all $p \in$ Prop,

- $\Pi ! \varphi:=\left\{X \cap \llbracket \varphi \rrbracket^{\mathbb{M}} \mid X \in \Pi\right\}-\{\emptyset\}$.

Finally, we can provide the model updated by issue-raising. Given $\mathbb{M}=$ $\langle W, R, \mu, \sigma, V, \Pi\rangle$, we define the $S P$-model $\mathbb{M} \# \varphi=\langle W \# \varphi, R \# \varphi, \mu \# \varphi, \sigma \# \varphi, V \# \varphi, \Pi \# \varphi\rangle$ as follows:

- $W \# \varphi:=W$, 
- $R \# \varphi:=R$,

- $(\mu \# \varphi):=\mu$,

- $(\sigma \# \varphi):=\sigma$,

- $(V \# \varphi):=V$,

- $(\Pi \# \varphi):=\left\{X \cap \llbracket \varphi \rrbracket^{\mathbb{M}}, X \cap \llbracket \neg \varphi \rrbracket^{\mathbb{M}} \mid X \in \Pi\right\}-\{\emptyset\}$.

Note that if $\mathbb{M}$ is an $S P$-model, then both $\mathbb{M} ! \varphi$ and $\mathbb{M} \# \varphi$ are themselves also $S P$-models. For $\mathbb{M} ! \varphi$ this basically follows from Dem15.7 Furthermore, it is very easy to see that the class of $S P$-models is also closed under issue-raising. Since the only difference between the original model $\mathbb{M}$ and the updated model $\mathbb{M} \# \varphi$ lies in their sixth components, we only need to check if $\Pi \# \varphi$ really is a partition on $W \# \varphi$. However, this is guaranteed by the definition of $\left.\Pi \# \varphi\right|^{8}$

The partition $\Pi$ is the crucial component of an $S P$-model. It is meant to keep track of the issues of epistemic interest. Informally, the idea is that a set $X \subseteq W$ is of epistemic interest iff it belongs to the partition $\Pi$ 9 By raising an issue, say the issue whether or not $\varphi$ is the case, one refines the partition $\Pi$ in such a way that there is no cell $C \in \Pi$ that intersects both $\llbracket \varphi \rrbracket^{\mathbb{M}}$ and $\llbracket \neg \varphi \rrbracket^{\mathbb{M}}$. This refined partition $\Pi \# \varphi$ will be the minimal refinement in the lattice of partitions satisfying this condition: $\Pi \# \varphi$ is the coarsest refinement of $\Pi$ such that for all $C \in \Pi$ it holds that $C \cap \llbracket \varphi \rrbracket^{\mathbb{M}}=\emptyset$ or $C \cap \llbracket \neg \varphi \rrbracket^{\mathbb{M}}=\emptyset$.

The issues of epistemic interest crucially interact with surprise. This becomes clear in the updated surprise intensity function $\sigma ! \varphi$. In Dem15], this was defined as follows: $(\sigma ! \varphi)(w)(X):=1-\mu(w)(X)$. This is essentially just a 'dynamified' version of the original insight that surprise is correlated with unexpectedness (cf. $S(X)=1-P(X)$ in Section 2). However, in the current framework, we do not just look at the probability of $X$ itself, but rather at the probability of its closure (relative to the partition $\Pi)$ :

$(\sigma ! \varphi)(w)(X):=1-\mu(w)(\Pi(X))$. Surprise about $X$ (elicited by the announcement of $\varphi$ ) is thus not inversely correlated with the probability of $X$ itself, but rather with the probability of the smallest issue of epistemic interest that contains $X$, i.e. $\Pi(X)$. We will see the effects of these definitions in Section 6 .

\footnotetext{
${ }^{7}$ In particular, note that $(\mu ! \varphi)(w)(v)$ is well-defined. After all, it holds that $w \in W ! \varphi=$ $\llbracket \varphi \rrbracket^{\mathbb{M}}$, and thus $\mu(w)\left(\llbracket \varphi \rrbracket^{\mathbb{M}}\right) \geq \mu(w)(w)$. But we required that $\mu(w)(w)>0$ in Definition 2 so we can conclude that $\mu(w)\left(\llbracket \varphi \rrbracket^{\mathbb{M}}\right)>0$.

8 As was already remarked earlier, our notion of raising an issue of epistemic interest is roughly similar to Lorini and Castelfranchi's LC07 operation of 'retrieving' a representation to put it under active scrutiny. This similarity applies in particular to both systems' dynamic modalities, but far less to their static operators. In particular, our [\# $\varphi]$-operator is the direct counterpart of their [retrieve $(\varphi)]$-operator. However, Lorini and Castelfranchi's static Test $(\varphi)$-operator does not have a direct counterpart in the present system.

${ }^{9}$ This bears some resemblance to the issue management system that van Benthem and Miniča use to study questions vBM12, and inquisitive semantics more generally [CGR19. One could even see issue-raising as a dynamic counterpart of awareness [FH87. It is important to note, however, that we will exploit the topological structure of such a semantics in combination with ideas from probability theory.
} 


\section{The Metatheory of $\mathcal{S U R \mathcal { P }} \mathcal{R} \mathcal{I} \mathcal{S}$ !}

We now turn to the metatheory of the logical system that was introduced in the previous section. We propose an axiomatization in Subsection 5.1. and prove that it is sound and complete in Subsection 5.2 .

\subsection{Axiomatization of $\mathcal{S U R \mathcal { R }} \mathcal{R} \mathcal{I} \mathcal{S E}$ !}

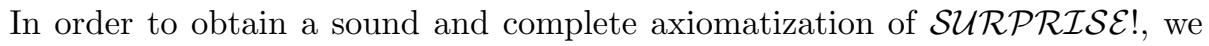
will first axiomatize the behavior of the dynamic operators, and then that of the static fragment (i.e. the fragment of $\mathcal{L}$ that does not contain any dynamic operators). Our strategy will be to express dynamic formulas in terms of logically equivalent static formulas by means of so-called reduction axioms, thus essentially reducing the full language $\mathcal{L}$ to its static fragment. Once this is done, proving soundness and completeness for the entire axiomatization of $\mathcal{S U R \mathcal { P }} \mathcal{R} \mathcal{I} \mathcal{E}$ ! will boil down to proving soundness and completeness of its static fragment.

The full list of reduction axioms can be found in Definition 5 . They essentially constitute an exhaustive case analysis of the BNF of $\mathcal{L}$, for both of the dynamic operators (public announcement and issue-raising). The two key axioms are those for the closure operator (under each of the dynamic operators). The soundness of these two axioms is established in Propositions 1 and 2 . First, however, it will be useful to prove two auxiliary lemmas, which allow us to express the closure of a set $X$ with respect to the updated partition (either $\Pi \# \varphi$ or $\Pi ! \varphi$ ) in terms of (closures with respect to) the original partition $\Pi$.

Lemma 1. (Closure lemma for \#) Given an $S P$-model $\mathbb{M}=\langle W, R, \mu, \sigma, V, \Pi\rangle$ and a formula $\varphi \in \mathcal{L}$, the following holds for any $X \subseteq W \# \varphi$ :

$$
(\Pi \# \varphi)(X)=\left(\Pi\left(X \cap \llbracket \varphi \rrbracket^{\mathbb{M}}\right) \cap \llbracket \varphi \rrbracket^{\mathbb{M}}\right) \cup\left(\Pi\left(X \cap \llbracket \neg \varphi \rrbracket^{\mathbb{M}}\right) \cap \llbracket \neg \varphi \rrbracket^{\mathbb{M}}\right) .
$$

Proof. Consider the following chain of equalities:

$$
\begin{aligned}
&(\Pi \# \varphi)(X)= \bigcup\{B \in \Pi \# \varphi \mid B \cap X \neq \emptyset\} \\
&=\left(\bigcup\left\{B \cap \llbracket \varphi \rrbracket^{\mathbb{M}} \mid B \cap \llbracket \varphi \rrbracket^{\mathbb{M}} \cap X \neq \emptyset, B \in \Pi\right\}\right) \\
& \cup\left(\bigcup\left\{B \cap \llbracket \neg \varphi \rrbracket^{\mathbb{M}} \mid B \cap \llbracket \neg \varphi \rrbracket^{\mathbb{M}} \cap X \neq \emptyset, B \in \Pi\right\}\right) \\
&=\left(\bigcup\left\{B \in \Pi \mid B \cap \llbracket \varphi \rrbracket^{\mathbb{M}} \cap X \neq \emptyset\right\} \cap \llbracket \varphi \rrbracket^{\mathbb{M}}\right) \\
& \cup\left(\bigcup\left\{B \in \Pi \mid B \cap \llbracket \neg \varphi \rrbracket^{\mathbb{M}} \cap X \neq \emptyset\right\} \cap \llbracket \neg \varphi \rrbracket^{\mathbb{M}}\right) \\
&=\left(\Pi\left(X \cap \llbracket \varphi \rrbracket^{\mathbb{M}}\right) \cap \llbracket \varphi \rrbracket^{\mathbb{M}}\right) \cup\left(\Pi\left(X \cap \llbracket \neg \varphi \rrbracket^{\mathbb{M}}\right) \cap \llbracket \neg \varphi \rrbracket^{\mathbb{M}}\right) .
\end{aligned}
$$


Lemma 2. (Closure lemma for !) Given an $S P$-model $\mathbb{M}=\langle W, R, \mu, \sigma, V, \Pi\rangle$ and a formula $\varphi \in \mathcal{L}$, the following holds for any $X \subseteq W ! \varphi$ :

$$
(\Pi ! \varphi)(X)=\Pi\left(X \cap \llbracket \varphi \rrbracket^{\mathbb{M}}\right) \cap \llbracket \varphi \rrbracket^{\mathbb{M}} .
$$

Proof. Analogous to the proof of Lemma 1

Proposition 1. For any $S P$-model $\mathbb{M}$ and possible world $w$, the following holds:

$$
\begin{gathered}
\mathbb{M}, w \models[\# \varphi] \operatorname{cl}(\psi) \\
\text { iff } \\
\mathbb{M}, w \models(\operatorname{cl}([\# \varphi] \psi \wedge \varphi) \wedge \varphi) \vee(\operatorname{cl}([\# \varphi] \psi \wedge \neg \varphi) \wedge \neg \varphi)
\end{gathered}
$$

Proof. Consider the following chain of equivalences:

$$
\begin{array}{ll}
\mathbb{M}, w \models[\# \varphi] c l(\psi) & \text { iff } \\
\mathbb{M} \# \varphi, w \models c l(\psi) & \text { iff } \\
w \in(\Pi \# \varphi)\left(\llbracket \psi \rrbracket^{\mathbb{M} \# \varphi}\right) & \text { iff } \\
w \in(\Pi \# \varphi)\left(\llbracket[\# \varphi] \psi \rrbracket^{\mathbb{M}}\right) & \text { iff } \\
w \in\left(\Pi\left(\llbracket[\# \varphi] \psi \rrbracket^{\mathbb{M}} \cap \llbracket \varphi \rrbracket^{\mathbb{M}}\right) \cap \llbracket \varphi \rrbracket^{\mathbb{M}}\right) & \\
\quad \cup\left(\Pi\left(\llbracket[\# \varphi] \psi \rrbracket^{\mathbb{M}} \cap \llbracket \neg \varphi \rrbracket^{\mathbb{M}}\right) \cap \llbracket \neg \varphi \rrbracket^{\mathbb{M}}\right) & \text { iff } \\
\mathbb{M}, w \models(c l([\# \varphi] \psi \wedge \varphi) \wedge \varphi) \vee(\operatorname{cl}([\# \varphi] \psi \wedge \neg \varphi) \wedge \neg \varphi) &
\end{array}
$$

Note that step (5) is justified by Lemma 1.

Proposition 2. For any $S P$-model $\mathbb{M}$ and any possible world $w$, the following holds 10

$$
\mathbb{M}, w \models[! \varphi] c l(\psi) \text { iff } \mathbb{M}, w \models \varphi \rightarrow \operatorname{cl}(\langle! \varphi\rangle \psi)
$$

Proof. Consider the following chain of equivalences:

$$
\begin{array}{ll}
\mathbb{M}, w \models[! \varphi] c l(\psi) & \text { iff } \\
\text { if } \mathbb{M}, w \models \varphi, \text { then } \mathbb{M} ! \varphi, w \models c l(\psi) & \text { iff } \\
\text { if } \mathbb{M}, w \models \varphi, \text { then } w \in(\Pi ! \varphi)\left(\llbracket \psi \rrbracket^{\mathbb{M} !}\right) & \text { iff } \\
\text { if } \mathbb{M}, w \models \varphi, \text { then } w \in(\Pi ! \varphi)\left(\llbracket\langle! \varphi\rangle \psi \rrbracket^{\mathbb{M}}\right) & \text { iff } \\
\text { if } \mathbb{M}, w \models \varphi, \text { then } w \in \Pi\left(\llbracket\langle! \varphi\rangle \psi \rrbracket^{\mathbb{M}} \cap \llbracket \varphi \rrbracket^{\mathbb{M}}\right) \cap \llbracket \varphi \rrbracket^{\mathbb{M}} & \text { iff } \\
\mathbb{M}, w \models \varphi \rightarrow(c l(\langle! \varphi\rangle \psi \wedge \varphi) \wedge \varphi) & \text { iff } \\
\mathbb{M}, w \models \varphi \rightarrow c l(\langle! \varphi\rangle \psi) &
\end{array}
$$

Note that step (11) is justified by Lemma 2

\footnotetext{
${ }^{10}$ For the sake of self-containment we add that $\langle! \varphi\rangle \psi$ is the dual of $[! \varphi] \psi$.
} 
We can now give the the full axiomatization of $\mathcal{S U R \mathcal { R }} \mathcal{R} \mathcal{I} \mathcal{E}$ !.

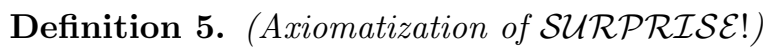

The static fragment of $\mathcal{S U R \mathcal { R }} \mathcal{R} \mathcal{I} \mathcal{S}$ ! is axiomatized by 1-6, while its dynamic fragment is axiomatized by 7-13.

1. All of propositional logic, including modus ponens,

2. the modal logic $S 5$ for $K$,

3. the axioms for linear inequalities from Fagin, Halpern and Megiddo FHM90, FH94]:

- $\left(a_{1} P\left(\varphi_{1}\right)+\cdots+a_{k} P\left(\varphi_{k}\right) \geq b\right) \leftrightarrow\left(a_{1} P\left(\varphi_{1}\right)+\cdots+a_{k} P\left(\varphi_{k}\right)+\right.$ $\left.0 P\left(\varphi_{k+1}\right) \geq b\right)$

- $\left(a_{1} P\left(\varphi_{1}\right)+\cdots+a_{k} P\left(\varphi_{k}\right) \geq b\right) \rightarrow\left(a_{j_{1}} P\left(\varphi_{1}\right)+\cdots+a_{j_{k}} P\left(\varphi_{k}\right) \geq b\right)$, if $j_{1}, \ldots, j_{k}$ is a permutation of $1, \ldots, k$,

- $\left(a_{1} P\left(\varphi_{1}\right)+\cdots+a_{k} P\left(\varphi_{k}\right) \geq b\right) \wedge\left(a_{1}^{\prime} P\left(\varphi_{1}\right)+\cdots+a_{k}^{\prime} P\left(\varphi_{k}\right) \geq b^{\prime}\right) \rightarrow$ $\left(\left(a_{1}+a_{1}^{\prime}\right) P\left(\varphi_{1}\right)+\cdots+\left(a_{k}+a_{k}^{\prime}\right) P\left(\varphi_{k}\right) \geq b+b^{\prime}\right)$,

- $\left(a_{1} P\left(\varphi_{1}\right)+\cdots+a_{k} P\left(\varphi_{k}\right) \geq b\right) \leftrightarrow\left(d a_{1} P\left(\varphi_{1}\right)+\cdots+d a_{k} P\left(\varphi_{k}\right) \geq d b\right)$ if $d>0$,

- $\left(a_{1} P\left(\varphi_{1}\right)+\cdots+a_{k} P\left(\varphi_{k}\right) \geq b\right) \vee\left(a_{1} P\left(\varphi_{1}\right)+\cdots+a_{k} P\left(\varphi_{k}\right) \leq b\right)$,

- $\left(a_{1} P\left(\varphi_{1}\right)+\cdots+a_{k} P\left(\varphi_{k}\right) \geq b\right) \rightarrow\left(a_{1} P\left(\varphi_{1}\right)+\cdots+a_{k} P\left(\varphi_{k}\right)>c\right)$ if $b>c$,

4. the Kolmogorov axioms for P [FH94]:

- $P(\varphi) \geq 0$,

- $P(\top)=1$,

- $P(\varphi \wedge \psi)+P(\varphi \wedge \neg \psi)=P(\varphi)$,

- $P(\varphi)=P(\psi)$ if $\vdash \varphi \leftrightarrow \psi$,

- $P(\perp)=0$,

5. Demey's axioms for $S(\cdot)$ [Dem15]:

- $S(\varphi) \geq 0$

- $S(\varphi) \leq 1$,

- $\neg\left(S(\varphi) \leq k \wedge S(\varphi) \geq k^{\prime}\right)$ for all $k<k^{\prime}$,

- $S(\varphi) \geq k \wedge S(\varphi) \leq k$,

- if $\vdash \varphi \leftrightarrow \psi$ then $\vdash S(\varphi) \geq c \leftrightarrow S(\psi) \geq c$,

- if $\vdash \varphi \leftrightarrow \psi$ then $\vdash S(\varphi) \leq c \leftrightarrow S(\psi) \leq c$,

6. the axioms for the closure operator, $\operatorname{cl}(\cdot)$ :

- $\operatorname{cl}(\perp) \rightarrow \perp$, 
- $\operatorname{cl}(\varphi \vee \psi) \leftrightarrow(\operatorname{cl}(\varphi) \vee \operatorname{cl}(\psi))$,

- if $\vdash \varphi \rightarrow \psi$ then $\vdash \operatorname{cl}(\varphi) \rightarrow \operatorname{cl}(\psi)$,

- $\operatorname{cl}(\operatorname{cl}(\varphi)) \rightarrow \operatorname{cl}(\varphi)$,

- $\varphi \rightarrow c l(\varphi)$,

- $\operatorname{cl}(\neg \operatorname{cl}(\neg \varphi)) \rightarrow \varphi$,

7. necessitation for public announcement: if $\vdash \psi$, then $\vdash[! \varphi] \psi$,

8. necessitation for issue-raising: if $\vdash \psi$, then $\vdash[\# \varphi] \psi$,

9. the reduction axioms for public announcement are as follows:

- $[! \varphi] p \leftrightarrow \varphi \rightarrow p$,

- $[! \varphi] \neg \psi \leftrightarrow \varphi \rightarrow \neg[! \varphi] \psi$

- $[! \varphi]\left(\psi_{1} \wedge \psi_{2}\right) \leftrightarrow[! \varphi] \psi_{1} \wedge[! \varphi] \psi_{2}$,

- $[! \varphi] K \psi \leftrightarrow \varphi \rightarrow[! \varphi] K \psi$,

- $[! \varphi] \sum_{i} c_{i} P\left(\psi_{i}\right) \leq c \leftrightarrow \varphi \rightarrow \sum_{i} c_{i} P\left(\langle! \varphi\rangle \psi_{i}\right)-c P(\varphi) \leq 0$,

- $[! \varphi] S(\psi) \leq c \leftrightarrow \varphi \rightarrow P(c l(\langle! \varphi\rangle \psi)) \geq 1-c$,

- $[! \varphi] S(\psi) \geq c \leftrightarrow \varphi \rightarrow P(c l(\langle! \varphi\rangle \psi)) \leq 1-c$,

- $[! \varphi] \operatorname{cl}(\psi) \leftrightarrow \varphi \rightarrow \operatorname{cl}(\langle! \varphi\rangle \psi)$,

10. the reduction axioms for issue raising are as follows:

- $[\# \varphi] p \leftrightarrow p$,

- $[\# \varphi] \neg \psi \leftrightarrow \neg[\# \varphi] \psi$,

- $[\# \varphi]\left(\psi_{1} \wedge \psi_{2}\right) \leftrightarrow[\# \varphi] \psi_{1} \wedge[\# \varphi] \psi_{2}$,

- $[\# \varphi] K \psi \leftrightarrow K[\# \varphi] \psi$,

- $[\# \varphi] \sum_{i} c_{i} P\left(\psi_{i}\right) \leq c \leftrightarrow \sum_{i} c_{i} P\left([\# \varphi] \psi_{i}\right) \leq c$,

- $[\# \varphi] S(\psi) \leq c \leftrightarrow S([\# \varphi] \psi) \leq c$,

- $[\# \varphi] S(\psi) \geq c \leftrightarrow S([\# \varphi] \psi) \geq c$,

- $[\# \varphi] \operatorname{cl}(\psi) \leftrightarrow(\operatorname{cl}([\# \varphi] \psi \wedge \varphi) \wedge \varphi) \vee(\operatorname{cl}([\# \varphi] \psi \wedge \neg \varphi) \wedge \neg \varphi)$.

It turns out that the closure operator $\operatorname{cl}(\cdot)$ can be seen as a modal $\diamond$-style operator. Indeed, the first three axioms of item 6 of Definition 5 together with propositional calculus and modus ponens make $\mathrm{cl}(\cdot)$ into a normal modal operator. Furthermore, adding the last three axioms from 6 make $\operatorname{cl}(\cdot)$ into an S5 operator. In particular, the first, second, fourth and fifth axiom of 6 are a straightforward logical formalization of the Kuratowski closure axioms, which are known to be an algebraic expression of the topological notion of closure KPHWZ08, MT44. As is well-known, topological spaces can be used to provide a semantics for S4-type modal operators. Adding the sixth axiom from 6 makes $c l(\cdot)$ into an S5-type $\diamond$-operator, as it is just the modal B-axiom in disguise. Topologically, this axiom expresses that all open sets are also closed, which holds for the the partition topology. 


\subsection{Soundness and Completeness}

Proving soundness of the axiomatization in Definition 5 is now almost trivial: it suffices to check that all reduction axioms as well as all the static axioms hold in all $S P$-models, and that the rules are validity-preserving. See Propositions 1 and 2 for two examples. In contrast, proving completeness is significantly more involved, so that is what we will focus on in this subsection.

The crucial difference between the static fragments of the original framework $\mathcal{S U R P} \mathcal{R} \mathcal{I} \mathcal{E}$ and the new framework $\mathcal{S U R \mathcal { P }} \mathcal{I} \mathcal{S} E$ ! resides in the closure operator, and the semantic apparatus that goes with it. Like Dem15] we will prove completeness by means of a filtrated canonical model construction; the hard part will consist in defining the partition component of the canonical model.

Consider a set of formulas $\Sigma$ that is finite (up to logical equivalence of formulas) and closed under subformulas, under negation and under $c l(\cdot)$. The filtrated canonical model $\mathbb{M}^{\Sigma}$ will have as its possible worlds all maximal consistent subsets of $\Sigma$, i.e. $W^{\Sigma}:=\{\Gamma \subseteq \mathcal{L} \mid \Gamma$ is a maximally consistent subset of $\Sigma\}{ }^{11}$ We now define a special equivalence relation $S^{\Sigma}$ on $W^{\Sigma}$; the partition that corresponds to this equivalence relation $S^{\Sigma}$ will be the canonical partition $\Pi^{\Sigma}$. The equivalence relation $S^{\Sigma}$ is defined as follows:

Definition 6. For all $\Gamma, \Delta \in W^{\Sigma}$ :

$$
S^{\Sigma}(\Gamma, \Delta): \Leftrightarrow \text { for all } \varphi \in \Sigma: \text { if } \neg \operatorname{cl}(\neg \varphi) \in \Gamma \text { then } \varphi \in \Delta .
$$

The relation $S^{\Sigma}$ can also be characterized in another, equivalent fashion:

$$
S^{\Sigma}(\Gamma, \Delta) \text { iff for all } \varphi \in \Sigma: \text { if } \varphi \in \Delta \text {, then } \operatorname{cl}(\varphi) \in \Gamma \text {. }
$$

We still have to prove that $S^{\Sigma}$ is in fact a real equivalence relation:

Proposition 3. $S^{\Sigma}$ is an equivalence relation on $W^{\Sigma}$.

Proof. We have to prove that $S^{\Sigma}$ is reflexive, symmetric and transitive. Here we only show the hard case: symmetry. Consider arbitrary $\Gamma, \Delta \in W^{\Sigma}$, and suppose that $S^{\Sigma}(\Gamma, \Delta)$; we now have to prove that $S^{\Sigma}(\Delta, \Gamma)$. Since $S^{\Sigma}(\Gamma, \Delta)$ we have that

$$
\forall \varphi \in \Sigma: \text { if } \neg \varphi \in \Delta, \text { then } \operatorname{cl}(\neg \varphi) \in \Gamma .
$$

We have to show that for all $\varphi \in \Sigma$ : if $\neg \varphi \in \Gamma$, then $\operatorname{cl}(\neg \varphi) \in \Delta$. So consider an arbitrary $\varphi \in \Sigma$ and assume that $\neg \varphi \in \Gamma$, we will show that $\operatorname{cl}(\neg \varphi) \in \Delta$. Since $\Delta$ is a maximally consistent subset of $\Sigma$, either $\neg \operatorname{cl}(\neg \varphi) \in \Delta$ or $\operatorname{cl}(\neg \varphi) \in \Delta$. Let us assume, towards a contradiction, that $\neg \operatorname{cl}(\neg \varphi) \in \Delta$. Since $S^{\Sigma}(\Gamma, \Delta)$ it follows that $\operatorname{cl}(\neg \operatorname{cl}(\neg \varphi)) \in \Gamma$. By the sixth axiom specified under item 6 of Definition 5 it follows that $\varphi \in \Gamma$. But now both $\varphi$ and $\neg \varphi$ are elements of $\Gamma$, which contradicts the consistency of $\Gamma$. We thus find that $\neg \operatorname{cl}(\neg \varphi) \notin \Delta$, and hence $\operatorname{cl}(\neg \varphi) \in \Delta$, which concludes the proof.

\footnotetext{
11 Note that $W^{\Sigma}$ is finite (up to logical equivalence of formulas), because $\Sigma$ itself is finite (up to logical equivalence), and applying negation or $c l(\cdot)$ twice (or more) does not yield any new formulas: $\neg \neg \varphi$ is equivalent to $\varphi$, and $\operatorname{cl}(\operatorname{cl}(\varphi))$ is equivalent to $\operatorname{cl}(\varphi)$.
} 
Since $S^{\Sigma}$ is an equivalence relation on $W^{\Sigma}$, we can use it to define equivalence classes: for every $\Gamma \in W^{\Sigma}$, put $[\Gamma]:=\left\{\Delta \in W^{\Sigma} \mid S^{\Sigma}(\Gamma, \Delta)\right\}$. We can now explicitly define the partition $\Pi^{\Sigma}$ on $W^{\Sigma}$ that is associated with the equivalence relation $S^{\Sigma}$ as follows: $\Pi^{\Sigma}:=\left\{[\Gamma] \mid \Gamma \in W^{\Sigma}\right\}$. If we unpack all the definitions, this boils down to:

$$
\Pi^{\Sigma}=\left\{\left\{\Delta \in W^{\Sigma} \mid \forall \varphi \in \Sigma: \text { if } \varphi \in \Delta, \text { then } \operatorname{cl}(\varphi) \in \Gamma\right\} \mid \Gamma \in W^{\Sigma}\right\} .
$$

We are now ready to define the notion of a canonical model for $\Sigma$, establish the truth lemma, and finally, prove soundness and completeness of $\mathcal{S U} \mathcal{R} \mathcal{P} \mathcal{R} \mathcal{I} \mathcal{E} !$

Definition 7. (Canonical model) The canonical model $\mathbb{M}^{\Sigma}$ for $\Sigma$ is an SPmodel $\left\langle W^{\Sigma}, R^{\Sigma}, \mu^{\Sigma}, \sigma^{\Sigma}, V^{\Sigma}, \Pi^{\Sigma}\right\rangle$. The first five components of this tuple are taken directly from [Dem15], while $\Pi^{\Sigma}$ is the partition that corresponds to the equivalence relation $S^{\Sigma}$, as has just been discussed.

Lemma 3. (Truth Lemma) For all $\varphi \in \Sigma$ it holds that $\mathbb{M}^{\Sigma}, \Gamma \models \varphi$ iff $\varphi \in \Gamma$.

Proof. We only need to prove the case in which $\varphi$ is of the form $\operatorname{cl}(\varphi)$; all other cases are the same as in Dem15. Proving that

$$
\mathbb{M}^{\Sigma}, \Gamma \models \operatorname{cl}(\varphi) \text { iff } c l(\varphi) \in \Gamma
$$

amounts to proving that

$$
\left(\exists \Delta \in W^{\Sigma}: S^{\Sigma}(\Gamma, \Delta) \text { and } \varphi \in \Delta\right) \text { iff } c l(\varphi) \in \Gamma .
$$

left-to-right Suppose there exists some $\Delta \in W^{\Sigma}$ such that $S^{\Sigma}(\Gamma, \Delta)$ and $\varphi \in$ $\Delta$. By definition of $S^{\Sigma}$ it immediately follows that $\operatorname{cl}(\varphi) \in \Gamma$.

right-to-left Suppose that $\operatorname{cl}(\varphi) \in \Gamma$. We need to show that $\exists \Delta \in W^{\Sigma}$ : $S^{\Sigma}(\Gamma, \Delta)$ and $\varphi \in \Delta$. Let us first define the set $X$ as follows:

$$
X:=\{\neg \alpha \mid \neg \operatorname{cl}(\alpha) \in \Gamma\}
$$

In Lemma 4, it is shown that $X \cup\{\varphi\} \subseteq \Sigma$ is consistent. By the Lindenbaum lemma, there exists a $\Delta \in W^{\Sigma}$ that contains $X \cup\{\varphi\}$. It trivially follows that $\varphi \in \Delta$. To show that $S^{\Sigma}(\Gamma, \Delta)$, we need to show that for all $\psi \in \Sigma$ : if $\psi \in \Delta$, then $\operatorname{cl}(\psi) \in \Gamma$. We will show this by contradiction. Let us assume that there is some $\psi \in \Delta$ such that $\operatorname{cl}(\psi) \notin \Gamma$. Because of the maximality of $\Gamma$ this entails that $\neg \operatorname{cl}(\psi) \in \Gamma$. By definition of $X$, it follows that $\neg \psi \in X \subseteq \Delta$. But then both $\neg \psi \in \Delta$ and $\psi \in \Delta$, which contradicts the consistency of $\Delta$.

Lemma 4. The set $X \cup\{\varphi\}$ (as introduced in the previous proof) is consistent. 
Proof. Let us suppose, towards a contradiction, that $X \cup\{\varphi\}$ is inconsistent. This entails that $X \vdash \neg \varphi$, and hence there exist $\alpha_{1}, \ldots, \alpha_{N} \in X$ such that

$$
\alpha_{1}, \ldots, \alpha_{N} \vdash \neg \varphi .
$$

By definition of $X$, these $\alpha_{i}$ are all of the form $\neg \beta_{i}$, where $\neg \operatorname{cl}\left(\beta_{i}\right) \in \Gamma$. This means that

$$
\neg \beta_{1}, \ldots, \neg \beta_{N} \vdash \neg \varphi .
$$

By means of some propositional reasoning it follows that

$$
\varphi \vdash \beta_{1} \vee \cdots \vee \beta_{N} .
$$

Using the third inference rule specified under item 6 of Definition 5 , we get

$$
\operatorname{cl}(\varphi) \vdash \operatorname{cl}\left(\beta_{1} \vee \cdots \vee \beta_{N}\right) .
$$

We can apply one of the Kuratowski axioms:

$$
\operatorname{cl}(\varphi) \vdash \operatorname{cl}\left(\beta_{1}\right) \vee \cdots \vee \operatorname{cl}\left(\beta_{N}\right) .
$$

Since $\operatorname{cl}(\varphi) \in \Gamma$ and $\Gamma$ is maximal it follows that there is at least one $\beta_{k}$ such that $c l\left(\beta_{k}\right) \in \Gamma$. But recall that for all $\beta_{i}$ we have $\neg c l\left(\beta_{i}\right) \in \Gamma$. Hence, both $\neg c l\left(\beta_{k}\right)$ and $\operatorname{cl}\left(\beta_{k}\right)$ are elements of $\Gamma$, which contradicts the consistency of $\Gamma$.

Theorem 1. SURPRISE! is sound and (weakly) complete with respect to all SP-models.

Proof. We prove soundness in the traditional fashion: by induction on derivation complexity. For most axioms and derivation rules, we can rely on Dem15. For the reduction axioms of the $\operatorname{cl}(\cdot)$-operator under issue-raising and public announcement, see Propositions 1 and 2, respectively. The only cases we still have to deal with, are necessitation for issue-raising, the Kuratowski closure axioms and axiom 13 from Definition 5. These can all be established through easy semantic reasoning and topological arguments.

As for (weak) completeness, assume that $\not \varphi \varphi$; we will show that $\not \models \varphi$. First of all, because of the reduction axioms, $\varphi$ can be rewritten without any dynamic operators, i.e. there exists a formula $\varphi^{\prime} \in \mathcal{L}$ that does not contain any dynamic operators, such that $\vdash \varphi \leftrightarrow \varphi^{\prime}{ }^{12}$ Since $\forall \varphi$, it follows that $\forall \varphi^{\prime}$, and hence the set $\left\{\neg \varphi^{\prime}\right\}$ is consistent. Let $\Sigma \subseteq \mathcal{L}$ be the smallest set that contains $\neg \varphi^{\prime}$ and that is closed under subformulas as well as under negation and $\operatorname{cl}(\cdot)$. Note that $\Sigma$ is finite (up to logical equivalence of formulas), because $\neg \varphi$ has only finitely many distinct subformulas, and applying negation or $\operatorname{cl}(\cdot)$ twice does not yield any new formulas (cf. Footnote 11). Since $\left\{\neg \varphi^{\prime}\right\}$ is a consistent subset of $\Sigma$, it follows by means of the Lindenbaum lemma that there exists a maximal consistent subset $\Delta$ of $\Sigma$ such that $\left\{\neg \varphi^{\prime}\right\} \subseteq \Delta$, and hence $\neg \varphi^{\prime} \in \Delta$. By means of Lemma 3 it follows that $\mathbb{M}^{\Sigma}, \Delta \models \neg \sigma^{\prime}$. This entails that $\forall \varphi^{\prime}$. Now, since $\vdash \varphi \leftrightarrow \varphi^{\prime}$, it follows by soundness that also $=\varphi \leftrightarrow \varphi^{\prime}$, which together with $\not \models \varphi^{\prime}$ entails that $\not \models \varphi$.

\footnotetext{
${ }^{12}$ This can be shown using a translation argument, cf. vDvdHK07, 186-189].
} 
As a bonus we get that $\mathcal{S U R \mathcal { R }} \mathcal{R} \mathcal{I} \mathcal{S}$ ! has the finite model property.

Corollary 1. SURP $\mathcal{R} \mathcal{I S E}$ ! has the finite model property.

Proof. This follows directly from the construction above, since the canonical model $\mathbb{M}^{\Sigma}$ is, by definition, finite.

\section{The Expressive Power of $\mathcal{S U R \mathcal { P }} \mathcal{R} \mathcal{I} \mathcal{E}$ !}

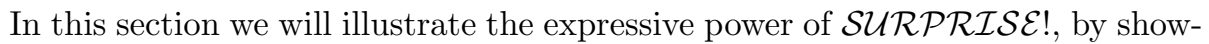
ing how this system can naturally capture a broad range of interesting scenarios. In particular, we will show that this system is capable of handling cases where Demey's system breaks down (cf. Section 2). Finally, we will argue that even though our system obeys a kind of 'garbage in garbage out' principle, this need not be a bad thing.

We begin by considering the original ball-picking scenario from Section 2

Example 1. (Randomly picking a ball from a bin) I randomly pick a ball from a bin filled with 10000 numbered balls. The ball I draw has the number 4592 printed on it. Intuitively, this does not elicit any surprise.

Recall that the original $\mathcal{S U R \mathcal { P }} \mathcal{R} \mathcal{I S E}$ system is unable to deal with such a scenario. By contrast, the new system is perfectly equipped to deal with it. Define an $S P$-model $\mathbb{M}:=\langle W, R, \mu, \sigma, V, \Pi\rangle$, where $W:=\left\{w_{1}, \ldots, w_{10000}\right\}$, $R:=W \times W, \mu(w)$ is the uniform probability mass function on $W, \sigma$ is irrelevant, $V\left(p_{i}\right)=\left\{w_{i}\right\}$ (where $p_{i}$ is the proposition that ball $i$ is picked, and $w_{i}$ is the possible world in which ball $i$ is picked, for $1 \leq i \leq 10000)$, and finally, $\Pi$ is the coarsest possible partition on $W$, i.e. $\Pi:=\{W\}$. One can now easily show that this model perfectly captures our intuitive assessment of the above scenario. In particular, we have

$$
\mathbb{M}, w_{4592} \models P\left(p_{4592}\right)=\frac{1}{10000} \wedge \quad\left[!_{4592}\right]\left(P\left(p_{4592}\right)=1 \wedge S\left(p_{4592}\right)=0\right) .
$$

Informally: initially (i.e. before any ball has been picked), the agent assigns probability $\frac{1}{10000}$ to ball 4592 being picked (just like she does for any other ball being picked); after ball 4592 has effectively been picked, she becomes certain about this and updates her probability to 1 (through Bayesian updating), but she does not experience any surprise about it (despite its initial unexpectedness). In order to verify the crucial part, i.e. [! $\left.p_{4592}\right] S\left(p_{4592}\right)=0$, consider the following 
chain of equivalences:

$$
\begin{array}{ll}
\mathbb{M}, w_{4592} \models\left[! p_{4592}\right] S\left(p_{4592}\right)=0 & \text { iff } \\
\text { if } \mathbb{M}, w_{4592} \models p_{4592}, \text { then } \mathbb{M} ! p_{4592}, w_{4592} \models S\left(p_{4592}\right)=0 & \text { iff } \\
\text { if } \mathbb{M}, w_{4592} \models p_{4592}, \text { then }\left(\sigma ! p_{4592}\right)\left(w_{4592}\right)\left(\llbracket p_{4592} \rrbracket^{\mathbb{M} ! p_{4592}}\right)=0 & \text { iff } \\
\text { if } \mathbb{M}, w_{4592} \models p_{4592}, \text { then }\left(\sigma ! p_{4592}\right)\left(w_{4592}\right)\left(\llbracket p_{4592} \rrbracket^{\mathbb{M}}\right)=0 & \text { iff } \\
\text { if } \mathbb{M}, w_{4592} \models p_{4592}, \text { then } 1-\mu\left(w_{4592}\right)\left(\Pi\left(\llbracket p_{4592} \rrbracket^{\mathbb{M}}\right)\right)=0 & \text { iff } \\
\text { if } \mathbb{M}, w_{4592} \models p_{4592}, \text { then } 1-\mu\left(w_{4592}\right)\left(\Pi\left(\left\{w_{4592}\right\}\right)\right)=0 & \text { iff } \\
\text { if } \mathbb{M}, w_{4592} \models p_{4592}, \text { then } 1-\mu\left(w_{4592}\right)(W)=0 & \text { iff } \\
\text { if } \mathbb{M}, w_{4592} \models p_{4592}, \text { then } 1-1=0 & \checkmark
\end{array}
$$

Note that the crucial aspect of this chain is that we do not consider $\mu\left(w_{4592}\right)\left(\llbracket p_{4592} \rrbracket^{\mathbb{M}}\right)$, but rather $\mu\left(w_{4592}\right)\left(\Pi\left(\llbracket p_{4592} \rrbracket^{\mathbb{M}}\right)\right)$. In other words, we do not work with $\llbracket p_{4592} \rrbracket^{\mathbb{M}}$ itself, but rather with its closure relative to $\Pi$. Since $\Pi$ is the coarsest possible partition on $W$, it has a very drastic effect: instead of looking at the singleton $\llbracket p_{4592} \rrbracket^{\mathbb{M}}=\left\{w_{4592}\right\}$ (which has probability $\frac{1}{10000}$ and would thus yield the intuitively incorrect surprise intensity $\frac{9999}{10000}$ ), we rather look at the entire set $\Pi\left(\llbracket p_{4592} \rrbracket^{\mathbb{M}}\right)=W$ (which has probability 1 and thus yields the intuitively correct surprise intensity 0 ).

The fact that we work with the coarsest possible partition on $W$ means that there are no genuine issues of epistemic interest. In the above scenario, all issues are equally interesting (or equivalently, equally bland): no proposition is deemed more interesting or worthy of investigation than any other. This changes drastically when we move to the variation on the ball-picking scenario that was also introduced in Section 2 .

Example 2. (Randomly picking a 'pre-specified' ball from a bin) I am about to randomly pick a ball from a bin filled with 10000 numbered balls. Another person states that she believes that ball 4592 will be picked. Next, I effectively draw a ball, which indeed turns out to be ball 4592. Intuitively, I am very surprised by this.

Recall, once again, that the original $\mathcal{S U R \mathcal { P R I S E }}$ system is unable to differentiate between this scenario and the previous one. By contrast, the new

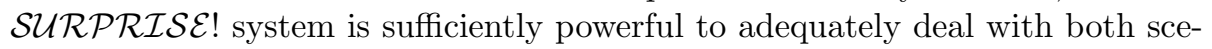
narios. Consider the same $S P$-model $\mathbb{M}$ as before. One can now easily show that this model perfectly captures our intuitive assessment of the second scenario as well. In particular, we have

$$
\begin{aligned}
& \mathbb{M}, w_{4592} \models P\left(p_{4592}\right)=\frac{1}{10000} \wedge \\
& {\left[\# p_{4592}\right]\left(P\left(p_{4592}\right)=\frac{1}{10000} \wedge\left[! p_{4592}\right]\left(P\left(p_{4592}\right)=1 \wedge S\left(p_{4592}\right)=\frac{9999}{10000}\right)\right) .}
\end{aligned}
$$

Informally: initially (i.e. before anything has happened), the agent assigns probability $\frac{1}{10000}$ to ball 4592 being picked (just like she does for any other 
ball being picked). By saying that she believes that ball 4592 will be picked, the other person raises the issue whether $p_{4592}$; this issue-raising does not have any immediate effects; in particular, the agent still assigns the same probability $\frac{1}{10000}$ to ball 4592 being picked. However, finally, after ball 4592 has effectively been picked, the agent not only updates her probability to 1 , but she also experiences a very high degree of surprise. In order to verify the crucial part, i.e. $\left[\# p_{4592}\right]\left[! p_{4592}\right] S\left(p_{4592}\right)=\frac{9999}{10000}$, consider the following chain of equivalences:

$$
\begin{array}{lr}
\mathbb{M}, w_{4592}=\left[\# p_{4592}\right]\left[! p_{4592}\right] S\left(p_{4592}\right)=\frac{9999}{10000} & \text { iff } \\
\mathbb{M} \# p_{4592}, w_{4592}=\left[! p_{4592}\right] S\left(p_{4592}\right)=\frac{9999}{10000} & \text { iff } \\
\text { if } \mathbb{M} \# p_{4592}, w_{4592}=p_{4592}, \text { then } \mathbb{M} \# p_{4592} ! p_{4592}, w_{4592}=S\left(p_{4592}\right)=\frac{9999}{10000} & \text { iff } \\
\text { if } \mathbb{M} \# p_{4592}, w_{4592} \models p_{4592}, \text { then }\left(\left(\sigma \# p_{4592}\right) ! p_{4592}\right)\left(w_{4592}\right)\left(\llbracket p_{4592} \rrbracket^{\left.\mathbb{M} ! p_{4592} \# p_{4592}\right)=\frac{9999}{10000}}\right. & \text { iff } \\
\text { if } \mathbb{M} \# p_{4592}, w_{4592}=p_{4592}, \text { then }\left(\left(\sigma \# p_{4592}\right) ! p_{4592}\right)\left(w_{4592}\right)\left(\llbracket p_{4592} \rrbracket^{\mathbb{M}}\right)=\frac{9999}{10000} & \text { iff } \\
\text { if } \mathbb{M} \# p_{4592}, w_{4592} \models p_{4592}, \text { then } 1-\left(\mu \# p_{4592}\right)\left(w_{4592}\right)\left(\left(\Pi \# p_{4592}\right)\left(\llbracket p_{4592} \rrbracket^{\mathbb{M}}\right)\right)=\frac{9999}{10000} & \text { iff } \\
\text { if } \mathbb{M} \# p_{4592}, w_{4592} \models p_{4592}, \text { then } 1-\mu\left(w_{4592}\right)\left(\left(\Pi \# p_{4592}\right)\left(\left\{w_{4592}\right\}\right)\right)=\frac{9999}{10000} & \text { iff } \\
\text { if } \mathbb{M} \# p_{4592}, w_{4592} \models p_{4592}, \text { then } 1-\mu\left(w_{4592}\right)\left(\left\{w_{4592}\right\}\right)=\frac{9999}{10000} & \text { iff } \\
\text { if } \mathbb{M} \# p_{4592}, w_{4592} \models p_{4592}, \text { then } 1-\frac{1}{10000}=\frac{9999}{10000} & \checkmark
\end{array}
$$

Because of the issue-raising (due to the statement by the other person), the question whether $p_{4592}$ holds has become an issue of epistemic interest, and hence, the partition has been refined accordingly: we move from the original partition $\Pi=\{\emptyset, W\}$ to the new partition $\Pi \# p_{4592}=\left\{\llbracket p_{4592} \rrbracket^{\mathbb{M}}, \llbracket \neg p_{4592} \rrbracket^{\mathbb{M}}\right\}=$ $\left\{\left\{w_{4592}\right\}, W-\left\{w_{4592}\right\}\right\}$. Consequently, whereas the closure of $\left\{w_{4592}\right\}$ relative to the original partition $\Pi$ is the entire domain $W$ (and thus yields probability 1 and surprise intensity 0 ), the closure of this same set $\left\{w_{4592}\right\}$ with respect to the new, refined partition $\Pi \# p_{4592}$ is just the singleton $\left\{w_{4592}\right\}$ (and thus yields probability $\frac{1}{10000}$ and surprise intensity $\frac{9999}{10000}$ ). This clearly shows how surprise intensity is not only determined by unexpectedness, but also by epistemic relevance (has the proposition under consideration been raised as an issue or not?).

Let us give a more complex example with a large set of possible worlds, several issues and a formula that is not of the form $[\# \varphi][! \varphi] S(\varphi)=c$.

Example 3. (Stock broker) Consider a stock broker. Based on her understanding of the market, she believes it to be very unlikely that stock $A$ will close at higher than $10.00 \$$. Moreover, as time progresses, she also comes to believe that 
it is unlikely that stock B will hit $20.00 \$$ or lower. If either one of these events does happen, she will have to reconsider her insight in the dynamics of the market. A week later she learns that stock $A$ just closed at $11 \$$ and stock $B$ just closed at 18\$. Of course, the stock broker is very surprised by this.

Let us try to make this more formal. For the set of possible worlds we will take the pairs of ticker values between $0.01 \$$ and $100.00 \$$ for stock $\mathrm{A}$ and stock $\mathrm{B}$, respectively, i.e. $W:=\{(x ; y) \mid 0.01 \leq x, y \leq 100.00$ and $100 x, 100 y \in \mathbb{N}\}{ }^{13}$ We set $R:=W \times W$. The stock broker's probabilities are distributed as follows: for all $w$ it holds that

$$
\mu(w)((x ; y)):=\left\{\begin{array}{ll}
\frac{8}{10(10 \cdot 100)(80 \cdot 100)} & \text { if } x \leq 10 \text { and } y>20 \\
\frac{1}{10(10 \cdot 100)(20 \cdot 100)} & \text { if } x \leq 10 \text { and } y \leq 20 \\
\frac{8}{100(90 \cdot 100)(80 \cdot 100)} & \text { if } x>10 \text { and } y>20 \\
\frac{2}{100(90 \cdot 100)(20 \cdot 100)} & \text { if } x>10 \text { and } y \leq 20
\end{array} .\right.
$$

In other words $W$ is partitioned in four sets of equiprobable outcomes with $8000000,2000000,72000000$ and 18000000 possible worlds, respectively. The probabilities assigned by $\mu(w)(\cdot)$ to the respective sets of equiprobable outcomes are $0.8,0.1,0.08$ and 0.02 , for any $w \in W . \sigma$ is again irrelevant and for $\Pi$ we again take the coarsest partition possible: $\Pi:=\{W\}$. We also define atomic propositions $p_{x ; y}$ (with $(x ; y) \in W$ ), which informally mean that stocks A and B have hit values $x$ and $y$, respectively, so that for all $w$, it holds that

$$
V(w)\left(p_{x ; y}\right):=\{(x ; y)\} .
$$

Our stock broker holds the categorical beliefs that stock A will not close at higher than $10 \$$ and that stock B will not hit $20 \$$ or lower. We will denote the first proposition by $a$, in other words $V(w)(a):=\{(x ; y) \in W \mid x \leq 10\}$. The second proposition will be denoted by $b$, in other words $V(w)(b):=\{(x ; y) \in$ $W \mid y>20\}$. The model yields a plausible surprise intensity for the stock broker:

$$
\mathbb{M},(11 ; 18) \models[\# a][\# b]\left[! p_{11 ; 18}\right] S\left(p_{11 ; 18}\right)=0.98 \text {. }
$$

\footnotetext{
${ }^{13}$ Since there are $10^{4}$ possible values for stock $\mathrm{A}$ and $10^{4}$ possible values stock $\mathrm{B}$, there are $10^{8}$ possible worlds in $W$.
} 
This can easily be checked as follows:

$$
\begin{array}{lr}
\mathbb{M},(11 ; 18) \models[\# a][\# b]\left[! p_{11 ; 18}\right] S\left(p_{11 ; 18}\right)=0.98 & \text { iff } \\
\mathbb{M} \# a,(11 ; 18) \models[\# b]\left[! p_{11 ; 18}\right] S\left(p_{11 ; 18}\right)=0.98 & \text { iff } \\
\mathbb{M} \# a \# b,(11 ; 18) \models\left[! p_{11 ; 18}\right] S\left(p_{11 ; 18}\right)=0.98 & \text { iff } \\
\text { if } \mathbb{M} \# a \# b,(11 ; 18) \models p_{11 ; 18} \text {, then } \mathbb{M} \# a \# b ! p_{11 ; 18},(11 ; 18) \models S\left(p_{11 ; 18}\right)=0.98 & \text { iff } \\
\text { if } \mathbb{M},(11 ; 18) \models p_{11 ; 18} \text {, then }\left(((\sigma \# a) \# b) ! p_{11 ; 18}\right)\left(w_{11 ; 18}\right)\left(\llbracket p_{11 ; 18} \rrbracket^{\left.((\mathbb{M} \# a) \# b) ! p_{11 ; 18}\right)}\right)=0.98 & \text { iff } \\
\text { if } \mathbb{M},(11 ; 18) \models p_{11 ; 18} \text {, then }\left(((\sigma \# a) \# b) ! p_{11 ; 18}\right)\left(w_{11 ; 18}\right)\left(\llbracket p_{11 ; 18} \rrbracket^{\mathbb{M}}\right)=0.98 & \text { iff } \\
\text { if } \mathbb{M},(11 ; 18) \models p_{11 ; 18} \text {, then } 1-((\mu \# a) \# b)((11 ; 18))\left(((\Pi \# a) \# b)\left(\llbracket p_{11 ; 18} \rrbracket^{\mathbb{M}}\right)\right)=0.98 & \text { iff } \\
\text { if } \mathbb{M},(11 ; 18) \models p_{11 ; 18} \text {, then } 1-\mu((11 ; 18))(((\Pi \# a) \# b)(\{(11 ; 18)\}))=0.98 & \text { iff } \\
\text { if } \mathbb{M},(11 ; 18) \models p_{11 ; 18} \text {, then } 1-\mu((11 ; 18))\left(\llbracket \neg a \wedge \neg b \rrbracket^{\mathbb{M}}\right)=0.98 & \text { iff } \\
\text { if } \mathbb{M},(11 ; 18) \models p_{11 ; 18} \text {, then } 1-0.02=0.98 . & \checkmark
\end{array}
$$

Note that $(\Pi \# a) \# b)$ equals $\left\{\llbracket a \wedge b \rrbracket^{\mathbb{M}}, \llbracket a \wedge \neg b \rrbracket^{\mathbb{M}}, \llbracket \neg a \wedge b \rrbracket^{\mathbb{M}}, \llbracket \neg a \wedge \neg b \rrbracket\right\}^{\mathbb{M}}$. Since $\{(11 ; 18)\} \subset \llbracket \neg a \wedge \neg b \rrbracket^{\mathbb{M}}$, it holds that its closure relative to the partition topology induced by $(\Pi \# a) \# b)$ is exactly $\llbracket \neg a \wedge \neg b \rrbracket^{\mathbb{M}}$.

Does this assignment of probabilities square with the scenario we presented? Since the stock broker believes that stock A will not close at $10 \$$ or higher and that stock $B$ will not close at $20 \$$ or lower, she will naturally ascribe a higher total probability to the sets of outcomes that correspond to her beliefs, i.e. the issues $a$ and $b$, than to the sets that correspond to the negation of her beliefs. In the same vein, she will be less surprised if she is less convinced of her beliefs. That is to say that she assigns lower probabilities to $a$ and $b$. Another interesting feature of the framework is that the distribution of probability mass within each cell of $\Pi$ is irrelevant on both an informal and a formal level. For example, suppose that the probability mass in the cell corresponding to $\llbracket a \wedge b \rrbracket^{\mathbb{M}}$ were not homogeneously distributed; this would not make any difference to the calculations above.

On a more general level, this example is interesting for several other reasons: First of all, Example 3 nicely highlights that multiple issues can be raised in a sensible manner 14 Secondly, it contextualizes our logic with respect to other logical work on surprise. Mar10] characterizes surprise in terms of categorical beliefs. By relying on the closure of the issues to calculate surprise, we can bridge the gap between categorical beliefs and rational degrees of belief. In Example 3. we operationalized categorical beliefs as issues. The interaction with rational degrees of belief is achieved through the natural topological structure the issues induce. Previous logics did not allow for dynamic (categorical) belief revision and therefore the beliefs had to be hard coded into the examples.

Because issues of epistemic interest directly factor into surprise phenomena, they also become a potential source of cognitive errors. This occurs, for example,

\footnotetext{
${ }^{14}$ Thanks to an anonymous referee for drawing our attention to such cases of iterated issueraising.
} 
in the case of a red herring, which is a fallacy of relevance VEGJ $^{+} 13$, p. 178]. A red herring shifts attention away to something that is actually of no importance. This phenomenon can be modeled as raising a 'junk issue'.

Example 4. (Red herring) Consider a highly credulous person, who has been fooled into thinking that some numbers have a certain supernatural importance. For the sake of concreteness, say that these numbers are 3, 7, 12 and 666. The credulous person randomly picks a ball from a bin of 10000 numbered balls, and the ball turns out be number 666. The person is highly surprised about this, marveling about the supernatural powers that must have been at work while she was picking the ball!

Although this person is clearly less than fully rational, the $\mathcal{S U R \mathcal { P }} \mathcal{I} \mathcal{S E}$ ! framework is perfectly able to capture such a scenario. Consider the same $S P$ model $\mathbb{M}$ as before, and let $q$ be the proposition that one of the supernatural numbers is drawn, i.e. $V(q)=\left\{w_{3}, w_{7}, w_{12}, w_{666}\right\}$. One can now easily show that this model perfectly captures the third scenario as well. In particular, we have $\mathbb{M}, w_{666}=[\# q]\left[!_{666}\right] S\left(p_{666}\right)=\frac{9996}{10000}$. The crucial step in verifying this is that $\left.1-\mu\left(w_{666}\right)\left((\Pi \# q)\left(\left\{w_{666}\right\}\right)\right)=1-\mu\left(w_{666}\right)\left(\left\{w_{3}, w_{7}, w_{12}, w_{666}\right\}\right)\right)=$ $1-\frac{4}{10000}=\frac{9996}{10000}$. After all, although the question whether $q$ holds is actually of no epistemic interest whatsoever, the credulous person nevertheless considers it relevant, and therefore her partition has been refined from $\Pi$ to $\Pi \# q=$ $\left\{\llbracket q \rrbracket^{\mathbb{M}}, \llbracket \neg q \rrbracket^{\mathbb{M}}\right\}=\left\{\left\{w_{3}, w_{7}, w_{12}, w_{666}\right\}, W-\left\{w_{3}, w_{7}, w_{12}, w_{666}\right\}\right\}$.

The $\mathcal{S U R \mathcal { P R }} \mathcal{I} \mathcal{S} \mathcal{E}$ ! system thus obeys a kind of 'garbage in garbage out' principle. If the partition $\Pi$ contains 'junk issues' (which are actually not of any epistemic interest), then the system will make predictions regarding agents' surprise intensities that do not appear very rational (e.g. an agent being very surprised, when she should actually not be surprised at all). However, we take this to be a feature, rather than a bug, of our system. After all, scenarios such as Example 3 show that such sub-rational cases of surprise do indeed occur, and the $\mathcal{S U R \mathcal { P }} \mathcal{R} \mathcal{I} \mathcal{S}$ ! system allows us to pinpoint the 'roots' of such irrational behavior (viz. the agents' junk issues).

This highlights the tension between any logical system and the actual behavior of human agents. Consider the following example: even though syllogistics might have prescribed a sound system of reasoning to the Greeks, not all of them will have applied it faithfully. Humans oftentimes engage in fallacious reasoning, but it cannot be the aim of logicians to accurately model such mistakes. In the case of syllogistic reasoning, Aristotle led the way by elucidating what makes for good classic reasoning. Similarly, a good logic for surprise should be able to capture what is interesting about surprise (we claim it is unexpectedness combined with epistemic interest) without providing justification for irrational behavior. We can now complete the analogy with syllogistics: just as one can validly deduce a false conclusion from a false premise, so one can derive a red herring from a junk issue. In other words, our logic provides a way to understand how people can come to be surprised by epistemically uninteresting events that they erroneously deem to be interesting after all, without thereby rationalizing or justifying that assumption. 
The notion of 'red herrings' or 'junk issues' also raises an important question for the theoretical framework $\mathcal{S U R \mathcal { P }} \mathcal{R} \mathcal{I} \mathcal{S}$ ! itself. Can we assume that the issues of epistemic interest (i.e. the partition component in an $S P$-model) are always uniquely specified, and how can we discover what they are in a given scenario? At the level of abstraction in this paper, we assume that the issues of epistemic interest are simply given. However, there seem to be many sensible ways of determining what partitions should correspond to actual issues. For example, one could try to combine this logical system with a utility-theoretic perspective. One could then define issues as sets of possible worlds that have a particularly high or low utility, thus marking possibilities of great gain or loss.

\section{Concluding Remarks}

In this paper we have developed a new logical system, $\mathcal{S U R \mathcal { R }} \mathcal{R} \mathcal{I} \mathcal{E}$ !, for reasoning about surprise in (human and artificial) agents. The system is based on earlier psychological and formal work which focuses on the close connection between surprise intensity and unexpectedness, but it also emphasizes the importance of issues of epistemic interest for surprise phenomena. The surprise intensity elicited by an event is thus not only determined by its unexpectedness (did the agent assign a high or a low probability to it?), but also by its epistemic relevance (has it been raised as an epistemic issue or not?). This is a strengthening of Demey's original philosophical point regarding the dynamic nature of surprise [Dem15], because both unexpectedness and issue-raising are thoroughly dynamic phenomena.

On a technical level, we have established a sound and complete axioma-

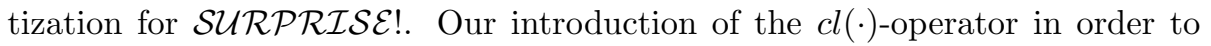
obtain a reduction axiom for $S$ under the dynamic [\#·]--operator is a nice example of 'language engineering', which is a well-known technique in the literature on dynamic epistemic logic. On a philosophical level, we have shown that $\mathcal{S U R \mathcal { P }} \mathcal{R} \mathcal{I} \mathcal{S E}$ ! is sufficiently expressive to capture a wide range of scenarios (many of which could not adequately be dealt with in earlier systems).

In future work, we aim to explore various extensions of the $\mathcal{S U R \mathcal { P }} \mathcal{R} \mathcal{I} \mathcal{E}$ ! system. For example, from a modeling perspective, it will be interesting to extend the framework from a single-agent to a multi-agent setting. This will allow us to capture an even wider range of scenarios, e.g. in which one agent is surprised that another agent is not surprised by some event. On a more technical level, we plan to investigate whether we obtain a useful notion of surprise if we take the interior, rather than the closure, relative to the partition topology in an $S P$-model.

\section{Acknowledgements}

We would like to thank Jan Heylen and Roger Vergauwen for their feedback on an earlier version of this paper. The first author is a $\mathrm{PhD}$ student at the G0B8616N project of the Research Foundation-Flanders (FWO), and the 
second author holds a Postdoctoral Fellowship of the Research FoundationFlanders (FWO). This paper presents the main results of the MA thesis of the first author, which was written in 2017-'18 under the supervision of the second author.

\section{References}

[BI05] Pierre Baldi and Laurent Itti. Attention: bits versus wows. In M. Zhao and Z. Shi, editors, International Conference on Neural Networks and Brain 2005 (ICNNEB'05), pages PL56-PL61. IEEE, 2005.

[BI10] Pierre Baldi and Laurent Itti. Of bits and wows: a Bayesian theory of surprise with applications to attention. Neural Networks, 23(5):649-666, 2010.

[CGR19] Ivano Ciardelli, Jeroen Groenendijk, and Floris Roelofsen. Inquisitive semantics. Oxford University Press, 2019.

[Cha64] William R. Charlesworth. Instigation and maintenance of curiosity behavior as a function of surprise versus novel and familiar stimuli. Child Development, 35(4):1169-1186, 1964.

[CL03] Cristiano Castelfranchi and Emiliano Lorini. Cognitive anatomy and functions of expectations. In Ron Sun, editor, IJCAI'03 Workshop on Cognitive Modeling of Agents and Multi-Agent Interactions. Morgan Kaufmann, 2003.

[Dem15] Lorenz Demey. The dynamics of surprise. Logique et Analyse, 58(230):251-277, 2015.

[DK15] Lorenz Demey and Barteld P. Kooi. Logic and probabilistic update. In Alexandru Baltag and Sonja Smets, editors, Johan van Benthem on Logical and Information Dynamics, pages 381-404. Springer, 2015.

[DS15] Lorenz Demey and Joshua Sack. Epistemic probabilistic logic. In Hans P. van Ditmarsch, Joseph Y. Halpern, Wiebe van der Hoek, and Barteld P. Kooi, editors, Handbook of Epistemic Logic, pages 147-202. College Publications, 2015.

[FH87] Ronald Fagin and Joseph Y Halpern. Belief, awareness, and limited reasoning. Artificial intelligence, 34(1):39-76, 1987.

[FH94] Ronald Fagin and Joseph Y Halpern. Reasoning about knowledge and probability. Journal of the ACM, 41(2):340-367, 1994. 
[FHM90] Ronald Fagin, Joseph Y Halpern, and Nimrod Megiddo. A logic for reasoning about probabilities. Information and Computation, 87(1-2):78-128, 1990.

[IB09] Laurent Itti and Pierre Baldi. Bayesian surprise attracts human attention. Vision Research, 49(10):1295-1306, 2009.

[KPHWZ08] Roman Kontchakov, Ian Pratt-Hartmann, Frank Wolter, and Michael Zakharyaschev. Topology, connectedness, and modal logic. In Carlos Areces and Robert Goldblatt, editors, Advances in Modal Logic, volume 7, pages 151-176. College Publications, 2008.

[LC07] Emiliano Lorini and Cristiano Castelfranchi. The cognitive structure of surprise: looking for basic principles. Topoi, 26(1):133-149, 2007.

[Mar10] Alexandru Marcoci. The surprise examination paradox in dynamic epistemic logic. M. Sc. thesis, University of Amsterdam, 2010.

[MC01] Luis Macedo and Amilcar Cardoso. Modeling forms of surprise in an artificial agent. In Johanna D. Moore and Keith Stenning, editors, Proceedings of the 23rd Annual Meeting of the Cognitive Science Society, pages 588-593. Lawrence Erlbaum Associates, 2001.

[MC12] Luís Macedo and Amílcar Cardoso. The exploration of unknown environments populated with entities by a surprise-curiositybased agent. Cognitive Systems Research, 19-20:62-87, 2012.

[MC17] Luis Macedo and Amílcar Cardoso. A Contrast-Based Computational Model of Surprise and Its Applications. Topics in Cognitive Science, 2017.

[MCRL09] Luis Macedo, Amilcar Cardoso, Rainer Reisenzein, and Emiliano Lorini. Artificial surprise. In Jordi Vallverdú and David Casacuberta, editors, Handbook of Research on Synthetic Emotions and Sociable Robotics: New Applications in Affective Computing and Artificial Intelligence, pages 267-291. IGI Global, 2009.

[MRC05] Luis Macedo, Rainer Reisenzein, and Amilcar Cardoso. Modeling forms of surprise in artificial agents: empirical and theoretical study of surprise functions. In Kenneth Forbus, Dedre Gentner, and Terry Regier, editors, Proceedings of the 26th Annual Meeting of the Cognitive Science Society, pages 873-838. Lawrence Erlbaum Associates, 2005.

[MT44] J.C.C. McKinsey and Alfred Tarski. The algebra of topology. Annals of Mathematics, 45(1):141-191, 1944. 
[OP87] Andrew Ortony and Derek Partridge. Surprisingness and expectation failure: what's the difference? In John McDermott, editor, Proceedings of the 10th International Joint Conference on Artificial Intelligence, pages 106-108. Morgan Kaufmann, 1987.

[Rei00] Rainer Reisenzein. The subjective experience of surprise. In Herbert Bless and Joseph P. Forgas, editors, The Message Within: The Role of Subjective Experience in Social Cognition and Behavior, pages 262-279. Taylor \& Francis, 2000.

[SPMR95] Joachim Stiensmeier-Pelster, Alice Martini, and Rainer Reisenzein. The role of surprise in the attribution process. Cognition 8 Emotion, 9(1):5-31, 1995.

[SS78] Lynn Arthur Steen and J. Arthur Seebach. Counterexamples in Topology. Springer, 1978.

[vBM12] Johan van Benthem and Ştefan Minică. Toward a dynamic logic of questions. Journal of Philosophical Logic, 41(4):633-669, 2012.

[vDvdHK07] Hans van Ditmarsch, Wiebe van der Hoek, and Barteld Kooi. Dynamic Epistemic Logic. Springer, 2007.

[VEGJ $\left.{ }^{+} 13\right]$ Frans H Van Eemeren, Rob Grootendorst, Ralph H Johnson, Christian Plantin, and Charles A Willard. Fundamentals of Argumentation Theory. Routledge, 2013. 